\title{
Manos a la Hoja: Un Taller de Escritura entre Jóvenes Sordos y Oyentes
}

\section{Manos a la Hoja: A Workshop on Writing between Deaf and Hearing Young People}

\author{
Miroslava Cruz-Aldrete ${ }^{1 *}$ \\ Miguel Ángel Villa Rodríguez ${ }^{2}$ \\ ${ }^{1}$ Universidad Autónoma del Estado de Morelos ${ }^{2}$ \\ Universidad Nacional Autónoma de México
}

\begin{abstract}
Se presenta la experiencia del taller "Manos a la hoja" realizado por un grupo de universitarios de la licenciatura en Letras Hispánicas de la Universidad Autónoma del Estado de Morelos con estudiantes sordos usuarios de la Lengua de Señas Mexicana (LSM) que cursan el bachillerato en CONALEP Morelos. Los objetivos eran, por un lado, el acercamiento entre los usuarios de distintas lenguas y de diferentes culturas en un mismo espacio educativo. Y, por el otro, ofrecer una respuesta desde la universidad a la demanda de una propuesta educativa para favorecer la competencia en la lengua escrita por parte del alumnado sordo inscrito en escuela regular. La definición de las actividades se basó en la reflexión de ambos grupos (sordos y oyentes) sobre la estructura gramatical de las lenguas de señas y las lenguas orales; los fines de la lengua escrita en la escuela; y el contacto entre lenguas y culturas.
\end{abstract}

Descriptores: Sordo, LSM, Lingüística aplicada, Fonología, Sintaxis.

In this paper we are talking about the workshop "Manos a la hoja". A group of university degree in Hispanic Literature at the Universidad Autónoma del Estado de Morelos work with a group deaf student's users Mexican Sign Language (LSM) at CONALEP Morelos's students. Aimed at, first, between users of different languages and different cultures in a single educational space. And, on the other, provide a response from the university, the demand for an educational proposal to promote competition in written by the deaf students enrolled in regular school language. The definition of the activities of the workshop was based on the reflection of both groups (deaf and hearing) of the grammatical structure of sign languages and spoken languages, and the purposes of the written language in school; and contact between people that use different languages and other cultures.

Keywords: Deaf, LSM, Applied linguistics, Phonology, Syntax. 


\section{Introducción}

Las cifras sobre el nivel de escolaridad que presentan las personas sordas en México resultan alarmantes. En el censo del 2010 reportado por el Instituto Nacional de Geografía y Estadística de México (INEGI, 2013) se menciona que el 35\% no tiene estudios, y solo el 5.4\% cursó algún grado de la educación media superior y el $4.1 \%$ de la superior. A su vez, la Organización para la Cooperación y el Desarrollo Económico (OCDE, 2013) revela que México tiene las tasas de matrícula más bajas entre los jóvenes de 15 a 19 años, puesto que cuatro de cada diez, no asisten a la escuela, y dentro de este grupo existe una mayor probabilidad que se encuentre a algún joven sordo.

Las posibles causas que pueden explicar este bajo nivel de escolaridad entre la comunidad sorda se encuentra el acceso a la educación bilingüe Lengua de Señas Mexicana (LSM)-español, la participación de intérpretes en la escuela, y el dominio del español escrito. Razones que en conjunto colocan a la juventud sorda usuaria de la lengua de señas en una condición de desventaja frente a su propio grupo generacional, pues ante este contexto muchos alumnos sordos quedan en principio fuera del esquema de educación básica (secundaria), y por tanto, es menos probable que continúen sus estudios.

Es notoria la baja competencia que presenta la mayoría de los alumnos sordos en el español en su forma escrita aun después de haber cursado el nivel básico (nueve o doce años de escolaridad). Situación que impacta en el acceso y permanencia del estudiante en el nivel educativo medio superior y superior, puesto que los alumnos sordos usuarios de la LSM ingresarán a escuelas regulares en donde la lengua de uso corriente es obviamente el español, y la comunidad educativa desconoce o no está sensibilizada al uso de la lengua de señas como la lengua natural de los sordos y les parece fácil suponer que el medio de comunicación tendrá que ser la lengua escrita, sin advertir que pasa el sordo es una segunda lengua.

Los alumnos sordos se enfrentan a una dinámica escolar que entre otras cosas les exige la participación en clase, la entrega de trabajos escritos, así como la resolución de exámenes, sin descartar las cuestiones administrativas que en ocasiones deben atenderse, y la interacción con sus pares de oyentes. Por tanto, contar con un intérprete en el aula, en el mejor de los casos, no es suficiente para desenvolverse adecuadamente en la escuela. Se requiere de un nivel adecuado de bilingüismo, LSM-español escrito, que permita a los jóvenes sordos responder a estas situaciones para desarrollarse en medio escolar regular de la mejor manera posible.

Al respecto, concordamos con el señalamiento de Pietrosemoli (2007) al discutir las exigencias del bilingüismo en relación con la comunidad sorda, quien pregunta qué nivel de competencia se exigiría al alumno cuya lengua materna no es la misma que se exige en el currículo escolar. Este pronunciamiento también coincide con la postura de otros investigadores al abordar el bilingüismo entre los usuarios de lenguas minoritarias y su relación con la lengua dominante en el contexto escolar (Paradis, Genesee y Crago, 2011), al identificar el papel de la lengua escrita (lengua dominante) como la lingua franca de la escuela. No solo por la posibilidad de acceder a través de ésta al conocimiento, sino porque es la lengua que está presente en todos los espacios de la vida escolar (señalamientos, avisos, etcétera). 
Sin embargo, el bilingüismo entre lenguas de la misma modalidad no comparte las mismas características, ni condiciones, que el bilingüismo entre lenguas cuya modalidad es diferente: lengua de señas-lengua oral, como se puede observar con los resultados obtenidos en el dominio de la lengua escrita, aun cuando en la actualidad contemos con la puesta en marcha del modelo bilingüe bicultural para la comunidad sorda.

La implementación del modelo bilingüe en el caso particular de la comunidad sorda de México, cuya operación está próxima a cumplir quince años, se enfrenta a dos situaciones adversas: la primera se relaciona con la adquisición tardía de la lengua de señas en la gran mayoría de los alumnos sordos; y la segunda, a la falta de recursos humanos (maestros oyentes competentes en LSM, maestros sordos) y materiales para la enseñanza.

Como mencionamos al inicio de nuestra discusión, una de las causas del bajo porcentaje del nivel de escolaridad de la comunidad sorda en nuestro país es la falta de un modelo educativo bilingüe que cubra las necesidades de este colectivo. Si bien, la evaluación de las formas de enseñanza de los alumnos sordos emanadas de este modelo es un asunto que requiere una investigación que rebasa los límites de este trabajo, consideramos necesario señalar este punto dado que los alumnos sordos que participaron en este estudio forman parte de una generación que se educó bajo los principios del bilingüismo (LSM-español), en los centros de atención múltiple (CAM) antes denominados escuelas de educación especial, y los resultados que arrojan su competencia en español nos llevan a reflexionar sobre la ejecución de este modelo bilingüe bicultural.

Por otra parte, no obviamos que el aprendizaje de la lengua escrita es una tarea compleja no solo para las personas sordas, sino también para las oyentes. Sin embargo pareciera que este hecho justifica que se simplifique la tarea a la que se enfrenta la comunidad sorda sin considerar que la lengua que se aprende no es su lengua materna. No se puede basar el aprendizaje del español de las personas sordas en los mismos supuestos que para los oyentes, pues la aparente transparencia del español se basa en la conciencia fonológica, en la discriminación del sonido y su correspondencia con la representación gráfica.

El dominio de la lengua escrita es un tema recurrente en las investigaciones sobre la educación del sordo a nivel mundial (Miller, 2010; Monreal y Hernández, 2005). Se ha discutido desde distintos puntos que van desde la competencia lingüística de la lengua de señas que presenta el alumno sordo, hasta la forma de enseñanza de la lengua dominante en su paso por el sistema de educación especial.

El objetivo de este trabajo es presentar una experiencia realizada por jóvenes (oyentes hablantes de español) de la licenciatura en Letras Hispánicas en la UAEM, con un grupo de jóvenes sordos usuarios de la LSM que cursan sus estudios en Colegio Nacional de Educación Profesional Técnica, CONALEP Morelos, en el taller denominado "Manos a la hoja" ${ }^{1}$ cuyo objetivo fue el abordaje de la enseñanza del español en su forma escrita con alumnos sordos que cursan el bachillerato, tomando como marco de referencia la lingüística aplicada a la enseñanza de segundas lenguas, y la pragmalingüística. De esta última rescatamos la idea que para conocer suficientemente una lengua, y lograr un

\footnotetext{
1 Este taller surge de uno de los programas de servicio social que se realiza en la Facultad de Humanidades de la Universidad Autónoma del Estado de Morelos (UAEM), coordinado por la Dra. Cruz-Aldrete.
} 
adecuado desarrollo lingüístico de la misma, es necesario conocer y adquirir rasgos de la cultura de la comunidad que la usa. Por tanto, este taller se basa en la interacción entre dos grupos de jóvenes con distintas lenguas y culturas, que comparten un mismo espacio escolar y definen metas comunes para la enseñanza-aprendizaje del español escrito.

Este documento se compone de dos secciones: en la primera parte discutimos el papel de la escuela como un espacio en el cual se puede favorecer o no el bilingüismo en los sordos. Esto a partir de la búsqueda de estrategias para la enseñanza de la lengua escrita a los jóvenes sordos usuarios de la LSM. En la segunda parte se describe la experiencia del taller "Manos a la hoja".

\section{La escuela: ¿un espacio para el bilingüismo?}

Desde hace varias décadas en México se ha creado una política lingüística y educativa encaminada a lograr un bilingüismo entre los usuarios de lenguas indígenas. Se busca que a partir de su ingreso a la escuela, comiencen el aprendizaje formal del español sin menoscabo de su lengua materna. No obstante, este supuesto bilingüismo empleado en la escuela ha sido duramente criticado y cuestionado su eficacia, dado que el ámbito escolar se convirtió, como ha señalado Coronado Suzán (1997), en un espacio prioritariamente monolingüe en el que se emplea solo la lengua dominante, el español. Se demostró que para muchos estudiantes indígenas la única lengua de instrucción y de comunicación entre ellos y los maestros era el español. De manera sistemática se discriminaba el uso de su lengua materna como el medio para la enseñanza y se enfatizaba el logro del dominio del español en su forma oral y escrita.

En la actualidad se puede observar que la mayoría de las comunidades indígenas presentan un grado de bilingüismo. Los hablantes recurren de manera diferenciada al uso de su lengua materna o del español, dependiendo de las situaciones de interacción social en que se encuentren. Por ejemplo, encontramos comunidades que restringen el uso de su lengua materna, al ámbito familiar o a las fiestas patronales; en cambio el uso del español gana otros espacios de la vida social, pues a través de esta lengua se realiza la comunicación con los "otros" en actividades de intercambio comercial, en el ámbito laboral, en la escuela, en el acceso a la salud, y en la impartición de la justicia.

Estas situaciones evidencian, por un lado, que en varias comunidades se reconoce al español como la lengua de prestigio provocando el desplazamiento de las lenguas originarias, y por el otro, se descubre que aun cuando se cuente con ambas lenguas para la interacción comunicativa, no significa que haya un aprendizaje y desarrollo homogéneo de los dos sistemas lingüísticos, ni el mismo grado de dominio, por parte de los usuarios, pues este dependerá entre otras cosas de la edad, del género y del rol del hablante al interior y al exterior de la comunidad.

Ser bilingüe no es opción para muchos miembros de comunidades lingüísticas minoritarias. Se convierte en una exigencia ante las circunstancias que viven en el ejercicio de sus derechos fundamentales (salud, educación, trabajo, justicia), y por tanto, se convierte en un fenómeno cuya complejidad nos conduce a la discusión de las estrategias a seguir para la enseñanza de la lengua dominante como segunda lengua sin menoscabo del valor de las lenguas originarias.

Ante esto, diversas instituciones se han dado a la tarea de crear talleres para documentar las lenguas originarias en los lugares donde aún se utilizan; y en el desarrollo de la 
Actividades que a su vez requieren de la participación de la comunidad con lo cual se fortalece el papel de los hablantes en el uso y conservación de su lengua materna.

El respeto de las minorías y de su lengua y cultura implica afrontar nuevos retos en la planificación de las políticas lingüísticas, en la implementaciónón de nuevas prácticas o modelos educativos en los cuales se privilegie el uso de la lengua materna de los educandos (Ayora Esteban, 2010; Terborg y García Landa, 2006), lo cual no excluye a las comunidades sordas usuarias de una lengua de señas.

Ahora bien, si contrastáramos las situaciones enunciadas con las condiciones que experimentan la comunidad sorda, sin duda encontraríamos varias similitudes. No obstante, el problema educativo se agudiza, no solo por el abordaje de la enseñanza del español escrito, sino por la adquisición de la LSM como su primera lengua. Muchos niños sordos llegan a las aulas sin haber estado expuestos a la lengua de señas, y con una baja competencia lingüística con respecto al español. Ambas condiciones impactan en su desarrollo integral y en su desempeño escolar.

Reconocemos que el modelo bilingüe ha favorecido la adquisición de la LSM entre los alumnos sordos de familias oyentes, sin embargo, no se ve el mismo resultado en el aprendizaje de la lengua escrita. Encontramos que las habilidades de la lectura y escritura en estos alumnos no son las esperadas. No corresponden al grado de escolaridad que cursan en la escuela regular.

Es probable que al abordar el tema de la enseñanza de la lengua escrita se parta del supuesto de que si los alumnos tienen consolidada la lengua de señas podrían desarrollar sin "mayores" problemas la literalidad en la lengua dominante. Hoy identificamos que si bien esta condición es la deseable no es la única para asegurar el aprendizaje del español escrito.

De tal modo, que después de más de una década de implementación de este modelo en México nos encontramos en un situación que nos conduce a preguntarnos si el bilingüismo en la escuela para sordos efectivamente se está desarrollando, o solo se trata de una aspiración que no se ha discutido de manera puntual, como han anotado numerosos autores al tratar la educación del sordo y la competencia en la lengua escrita (Lissi, Svartholm y González, 2012; Mayer y Akamatsu, 2003).

El resultado del taller propuesto Manos a la hoja demuestra que es urgente evaluar el modelo bilingüe y las expectativas que tiene la escuela al abrir las puertas para la inclusión educativa de los sordos sin considerar su dominio del español.

\subsection{El bilingüismo en la escuela para sordos: mito o realidad}

Existen numerosas investigaciones sobre el bilingüismo. La crítica que hace Ferreiro (1997) sobre este tema nos parece clave para nuestro estudio. Señala que al tratar cualquier aspecto del bilingüismo parece reconocerse que la situación ideal es el monolingüismo. Cuando se analiza el dominio real de las dos lenguas que tiene el hablante no se discute el grado de conciencia de los aspectos formales de las lenguas que entran en contacto. No se investiga cuál es el dominio formal de su lengua materna y sólo se evalúa su competencia en la lengua dominante. El señalamiento de Ferreiro debería ser una piedra angular al abordar la educación de los alumnos sordos dada la adquisición tardía de la LSM, y cómo bajo esta línea base se inicia el proceso de enseñanza aprendizaje del español escrito. 
La larga tradición que existe en México sobre la educación bilingüe instrumentada en las comunidades indígenas ha generado diversas investigaciones. Se ha puesto especial atención en el análisis de las condiciones y recursos de los estudiantes hablantes de una lengua originaria al enfrentarse a un medio escolarizado monolingüe en español; así como su acceso y permanencia en los distintos niveles educativos. Los textos de Hamel (2003, 2008) y Muñoz-Cruz (2010) constituyen un punto de referencia para entender la realidad educativa de estos alumnos; en ellos se analiza el tránsito de estos alumnos a los distintos niveles de educación (básica y superior).

Es posible hacer un parangón entre los hablantes de una lengua indígena y los sordos usuarios de la LSM. Ambos grupos pertenecen a una comunidad lingüística minoritaria y deben aprender el español de manera formal en la escuela durante los primeros años de su educación básica (primaria). No obstante esta comparación no resulta plausible en cuanto a la tarea de dominar una segunda lengua en su forma escrita. Aprender a leer y escribir no resulta de la misma complejidad dado el input lingüístico que han recibido al interior de sus casas y en la escuela (Schirmer, 2000; Schirmer y Williams 2003).

Si bien, hay una serie de elementos con los cuales todos los aprendices de la lengua escrita iniciamos, por ejemplo, conocer el alfabeto, reconocimiento de los signos de puntuación, direccionalidad de la escritura; hay otros procesos implicados, cognoscitivos y lingüísticos. Cualquier aprendiz no solo debe conocer el alfabeto, también debe saber usarlo, saber cómo se combinan esos elementos. Esto supone reconocer que hay un sistema de representación para las unidades de las lenguas naturales, que pueden ser utilizadas para escribir su lengua materna. Y, establecer una serie de hipótesis sobre cómo estas unidades constituyen la otra cara de la moneda de la producción (oral o de señas) de su lengua o de cualquier otra. Asimismo, debe atender a las reglas que subyacen a la organización de las distintas unidades que componen su discurso, y al uso de un sistema de puntuación. Y no menos importante, debe haber un interés por escribir.

Como se puede observar, el aprendizaje de la lengua escrita compromete una reflexión metalingüística. En el caso de los alumnos oyentes ésta se practica de manera sistemática desde edades tempranas, guiados por los profesores en las diferentes asignaturas y en todos los ciclos que conforman la educación básica. Desafortunadamente, esta actividad no se promueve con los alumnos sordos.

Es alarmante el vacío que existe en la formación de los alumnos sordos usuarios de la LSM sobre su propia lengua. Pues, a diferencia de otros grupos lingüísticos minoritarios educados bajo el esquema del bilingüismo (español-lengua originaria) donde sí se contempla en el currículo el estudio de su lengua materna, en el caso de la comunidad sorda no se ha diseñado un plan de estudios en donde se oferte un espacio para la enseñanza de la LSM.

Resulta lamentable que los alumnos sordos no estén desarrollando una reflexión metalingüística de su propia lengua, sin duda, esta actividad favorecería el aprendizaje de la lengua escrita, como podremos observar más adelante. Es una situación que refleja el análisis de Ferreiro (1997) al tratar el bilingüismo. Se hace un mayor hincapié o solo se considera el análisis formal de la lengua dominante, y no de la lengua materna del alumno.

\subsection{El estudio del aprendizaje y enseñanza de la lengua escrita para los sordos usuarios de las LS}


Entre las hipótesis que se han discutido para entender en dónde radican las dificultades para el aprendizaje de la lengua escrita (lectura y escritura) por parte de los alumnos sordos se encuentran la hipótesis fonológica y la hipótesis léxica. La primera relacionada con el déficit auditivo, considera que la falta de un adecuado input auditivo repercute en la falta de un óptimo desarrollo de la conciencia fonológica, que para algunos autores constituye un elemento básico para la lectura. Y la segunda, la hipótesis léxica, señala el acceso al almacén léxico que permite vincular el significado y el significante de la palabra escrita.

Abordar ambas hipótesis permite contextualizar algunos de los problemas que encontramos en las producciones escritas de los alumnos sordos. Pero, a partir de los datos obtenidos no es posible inclinarnos por alguna de ellas, no obstante, este primer acercamiento nos condujo a nuevas propuestas de investigación que corroboran o descartan cualquiera de estas hipótesis. Incluso abre un camino para ponderar los aportes de la pragmática sobre el uso de la lengua escrita

Desde la segunda mitad del siglo pasado (Blanton, Nunnaly y Odom, 1967; Hanson, 1989; Kavanagh, 1968; Kavanagh y Mattingly, 1972; Locke, 1978) se ha discutido que el habla (oral) es importante para la lectura, se destaca la relación entre el reconocimiento de las letras y el significado. Este hecho repercutió en la creación y ejecución de varios métodos para la enseñanza de la lectura y la escritura, los cuales se sustentan en el establecimiento de esta relación grafía-sonido. En el caso de la enseñanza del alumno sordo, entendemos por qué desde el oralismo este supuesto condujo a la práctica socorrida del adiestramiento auditivo, la lectura labio-facial, y la articulación, con el propósito de favorecer no solo el aprendizaje de la lengua oral sino también de la lengua escrita, pues se vinculaba la representación de dicho sonido (emitido y escuchado) con la grafía correspondiente.

La idea que los alumnos sordos podían acceder al conocimiento de la fonología a partir de eventos motores (articulación y lectura labio facial) implicados en el lenguaje fue discutida desde la década de los setenta del siglo pasado. Entre los estudios pioneros que se realizaron para demostrar la hipótesis fonológica en las personas sordas se encuentra el trabajo de Locke (1978), quien investigó si los sordos al igual que los oyentes se valían del apoyo fonológico para la lectura.

Locke trabajó con tareas de discriminación de pares mínimos; su estudio consistía en identificar una letra en dos contextos, en uno de ellos, la letra correspondía a un fonema típico asociado con el nombre de la letra, por ejemplo, "rag" o "rage". En cambio en el otro contexto la pronunciación de la letra no correspondía al fonema típico asociado al nombre de la grafía. A partir de esta diferenciación construyó un texto de 472 palabras con las cuales los sordos estaban familiarizados (de acuerdo con el profesor de lengua). A su vez se escogieron tres letras blanco que los participantes debían de identificar en el texto (c, g, h), las letras podían pertenecer a la modalidad fonémica o no fonémica. Y, por último, se consideró que la letra podía estar inserta en diferentes clases de palabras de orden mayor o menor.

En esta investigación participaron 24 sordos, estudiantes educados bajo el modelo oralista, cuyas edades se encontraban entre los 11 a 16 años. Y un grupo control integrado por 24 adolescentes oyentes de 12 a 13 años de edad, que asistían a una escuela pública regular. Se asignó a los participantes en forma aleatoria qué letra 
deberían identificar, y la tarea consistía en tachar en el texto la letra que se les había previamente indicado.

El análisis estadístico de los resultados de esta prueba demostró efectos significativos de la pronunciación (modal o no modal) y de la clase gramatical (nombre, verbo, adjetivo y palabra de función). Los participantes oyentes tuvieron un mayor efecto fonético y gramatical en comparación a los sordos. Se descubrió que había una mayor probabilidad de cometer errores cuando la letra que debían identificar no correspondía con un fonema asociado al nombre de la letra o bien no se pronunciaba. En cambio, en los sordos no hubo esta diferencia y había la misma probabilidad de cometer un error en cualesquiera de las modalidades.

Una situación similar sucedió con la clase gramatical; en los oyentes se diferenció la probabilidad de los errores en función de la clase gramatical. Así, había mayor probabilidad de errores en las palabras de función y menor en verbos, sustantivos y adjetivos. En contraste con el grupo de sordos cuya probabilidad del error fue prácticamente igual en todas las categorías gramaticales. Por tanto, esto condujo a suponer que los sordos perciben el texto escrito como estímulos visuales y no tanto como la representación gráfica de los fonemas.

Si partimos del supuesto de que la lengua escrita se construye como una representación de la fonología, la aparente transparencia del español con respecto a su relación grafíasonido hace que los docentes apuesten a una forma de enseñanza basada en la hipótesis fonológica. Pero, si bien hubo alumnos sordos que aprendieron de esta manera a leer y escribir, también hubo casos en los cuales los resultados no fueron satisfactorios, o al menos, no los esperados.

La representación fonológica es de naturaleza abstracta. El sistema lingüístico descansa en esta diferenciación entre unidades mínimas sin significado (fonemas), que al combinarse producen un cambio de significado, creando unidades de mayor alcance (con significado). Los hablantes nativos de cualquier lengua son capaces de segmentar estas unidades. Si bien el proceso de adquisición de la lengua materna se realiza de manera inconsciente, en los primeros años escolares los niños, guiados por el maestro, desarrollan la conciencia fonológica y por tanto pueden hacer una reflexión metalingüística sobre los elementos que constituyen su propia lengua.

$\mathrm{Al}$ respecto, nosotros creemos que los sordos presentan más dificultades para la lengua escrita (lectura y escritura) no solo porque su competencia fonológica puede ser limitada con respecto a la lengua dominante, sino porque no se ha propiciado en ellos una reflexión metalingüística de su propia lengua, la lengua de señas. No se discute con los alumnos que la LSM también tiene unidades mínimas que al combinarse forman unidades con significado (seña/palabra). Cómo esperar entonces que se desarrolle esta conciencia fonológica a partir del contacto con la segunda lengua sino se ha tenido la experiencia previa con la propia lengua materna.

Los estudios que apoyan la hipótesis fonológica nos conducen a proponer actividades que se relacionen con la percepción de las palabras (identificadas en la escritura por aparecer entre dos espacios blancos) en su totalidad, es decir, consideramos pertinente que los alumnos sordos tengan tareas que impliquen un barrido visual; la segmentación de palabras escritas considerando la frecuencia del patrón silábico (característico, en nuestro caso particular del español), pero, sin descartar su función dentro de una frase u 
oración. Si consideramos esto en su conjunto es posible que se propicie el establecimiento de diferentes redes de asociación que contribuyan a la formación de un concepto. Quizá así nos podamos acercar a la formación de un lexicón en una segunda lengua construido solo a partir de un input visual codificado a través del alfabeto.

Miller (2013) al abordar el fracaso lector en los sordos considera ambas hipótesis fonológica y léxica, en el sentido de una jerarquía de organización del sistema. Es decir, parte del conocimiento que éstos tienen sobre la estructura lingüística de la lengua que leen o escriben, desde la unidad mínima (fonema) hasta las unidades con significado y de mayor alcance (morfología y sintaxis).

Por un lado, supone que debido a la falta permanente de estimulación auditiva los sordos prelocutivos no desarrollan suficientemente la conciencia fonológica que les permitiría hacer una adecuada decodificación fonológica, y, consecuentemente, identificar las palabras escritas; en consecuencia fallan en el procesamiento sintáctico y semántico puesto que no pueden integrar el significado en niveles mayores de significado. Esta hipótesis tiene sentido en tanto que hay abundante evidencia que sugiere que las habilidades fonológicas de los sordos prelocutivos (conciencia fonológica, habilidades para la decodificación fonológica) están muy por debajo de su contraparte oyente (Charlier y Leybaert, 2000; Miller, 2006).

La otra hipótesis estudiada por Miller explica los problemas lectores de los sordos a partir de los déficits en el conocimiento estructural de la lengua, es decir, su poco entendimiento de la sintaxis no les permitiría hacer el enlace entre las palabras escritas y el campo más amplio de las ideas. Por tanto, se trataría de una alteración que atañe al nivel supraléxico de las oraciones. Se basa en el estudio que realizó con la participación de 62 sordos prelocutivos, de los cuales 36 eran estudiantes de educación media y 26 de licenciatura o posgrado. Los hallazgos arrojaron que las diferencias en la comprensión lectora no se explican por las habilidades metalingüísticas ni por la eficiencia en el procesamiento de palabras, sino por el procesamiento a un nivel supraléxico, al nivel de la frase.

En resumen, los ejemplos de las investigaciones aquí planteadas nos conducen a las siguientes conclusiones. Por un lado, la hipótesis fonológica no debería estar restringida al uso de los restos auditivos que pueden ser empleados por las personas sordas para la identificación de las grafías, sino en una reflexión metalingüística en la cual se considere que las palabras de una lengua se componen de elementos mínimos sin significado que al combinarse producen formas con significado, independientemente de su modalidad, oral o visogestual.

De igual manera, también habría que discutir sobre los diferentes alfabetos y formas de representar una lengua (caracteres chinos, el alfabeto cirílico o el alfabeto eurocéntrico, y el alfabeto manual) para establecer su vinculación con la lengua escrita de la lengua dominante.

Aunado a lo anterior, la hipótesis léxica nos conduce al análisis de las características gramaticales de la lengua de señas y de la lengua dominante. Identificando, semejanzas y diferencias al tratarse de lenguas de distinta modalidad. Y cómo la combinación de las unidades mínimas que compone cada lengua generan un sin fin de unidades con significado que componen el discurso. 
Para el análisis del aprendizaje de la lengua escrita, además de las hipótesis descritas, también recurrimos a un estudio de corte neuropsicológico. Así, en el 2012 realizamos una primera investigación con 30 estudiantes del plantel Temixco del Colegio Nacional de Educación Técnica (CONALEP, institución educativa del nivel Medio Superior que forma parte del Sistema Nacional de Educación Tecnológica) del Estado de Morelos, quienes cursaban el primer año de la carrera de alimentos y bebidas. Este plantel con una mirada inclusiva conformó algunos grupos por alumnos sordos y oyentes, apoyados por un intérprete en LSM-español que acompañaba al grupo en todas sus actividades.

El grupo que participó en esta investigación estaba integrado por 11 alumnos sordos usuarios de la LSM, y 19 alumnos oyentes hablantes de español. Todos los sujetos fueron evaluados en una sola sesión que duraba entre 30 y 45 minutos. Las instrucciones a los alumnos sordos eran dadas por un intérprete de LSM con quien se acordó previamente cómo tenían que darse las instrucciones para no proporcionar más información de la establecida en el manual de aplicación.

La selección de pruebas (ampliamente utilizadas en la evaluación neuropsicológica a nivel internacional) tenían como objetivo evaluar las habilidades cognitivas básicas: a) atención visual; b) velocidad de procesamiento; c) memoria de trabajo; d) memoria visual de corto y largo plazos; e) aprendizaje incidental; f) percepción; g) acceso a la semántica visual y léxica; $y$ h) funciones ejecutivas. Las pruebas seleccionadas fueron: cubos de Corsi (Milner, 1971), test de la figura compleja (Osterrieth, 1944; Rey, 1941), test de pirámides y palmeras (Howard y Patterson, 1992), y dígitos y símbolos claves del test Barcelona (Peña-Casanova, 1991).

Entre los resultados obtenidos encontramos que no hubo diferencias significativas entre las medias de los puntajes obtenidos por ambos grupos, excepto en dos tareas: cubos de Corsi ante la tarea de señalar los cubos en orden inverso que es una tarea en la que está implicada la memoria de trabajo; y en la segunda parte del test de pirámides y palmeras en la que tenían que realizar la tarea semántica a partir de las palabras escritas y no de las imágenes como en la primera parte.

Ambos resultados nos condujeron a reconocer una diferencia significativa en la memoria de trabajo y a suponer que esta función cognitiva puede estar en la base de la reconocida dificultad para el aprendizaje de la lectura. Así, aun cuando uno podría pensar que dada la naturaleza viso gestual de las lenguas de señas, los sordos señantes deberían tener un mayor desarrollo de las habilidades visoespaciales, esto no fue así. Nuestros datos no confirman esta suposición y marcan las diferencias en el procesamiento cognitivo on line de la información viso espacial. Es probable que en la base de las marcadas dificultades que presentan para el aprendizaje de esta habilidad, se encuentre una diferencia significativa en el procesamiento cognitivo de la información visual y que tenga que ver con la memoria de trabajo.

La discusión sobre las hipótesis fonológica, léxica y sobre la memoria de trabajo, permitió definir qué se haría con respecto a la enseñanza del español como segunda lengua, considerando además los aportes de la lingüística aplicada. 


\section{El taller "Manos a la hoja": un espacio bilingüe}

La propuesta de intervención realizada en el 2014, que dio origen al taller "Manos a la hoja" tenía como fin desarrollar una propuesta de enseñanza la lectura y escritura para jóvenes sordos señantes a partir del estudio de la gramática del español, coordinado por los jóvenes con lenguas y culturas distintas. Participaron alumnos oyentes de la carrera en Letras Hispánicas quienes diseñaron estrategias para la enseñanza del español como segunda lengua.

Este taller se desarrolló durante el segundo semestre del 2014 en CONALEP Morelos, en las instalaciones de CONALEP Temixco y CONALEP Cuautla. Participaron nueve alumnos de la UAEM del séptimo semestre de la licenciatura en Letras Hispánicas, participantes del programa de servicio social Iniciación a la investigación Lingüística de la LSM y la Comunidad Sorda, y 22 alumnos sordos usuarios de la LSM de la carrera técnica de Alimentos y bebidas. Una vez a la semana se impartía el taller en cada uno de los planteles mencionados, con una duración de dos horas, después de haber concluido el horario de sus clases curriculares. La asistencia al taller era de manera voluntaria.

Si bien, todos los alumnos sordos de este grupo (en su mayoría diagnosticados con sordera profunda) se identifican con el uso de la LSM, presentaban distintos niveles de competencia en la lengua de señas. Del total del grupo, 17 de los alumnos que provenían de centros de atención múltiple con un enfoque bilingüe mostraban mayor dominio de la LSM, de ese grupo cuatro mujeres y un hombre, eran considerados al interior del colectivo como aquellos que realmente conocían la LSM. Tres de los alumnos, provenían de escuelas regulares, habían pasado por años de terapia de lenguaje, y comenzaban a partir de su ingreso a CONALEP, y al entrar en contacto con sus pares sordos a adquirir la LSM. Dos de los alumnos presentaban una hipoacusia media, con el empleo del auxiliar auditivo podían alcanzar a escuchar cierto rango de frecuencias de los sonidos del lenguaje y, quizá, por ello, preferían que las explicaciones fueran de manera oral y no por señas. Ninguno de los alumnos era hijo de padres sordos, todos provenían de hogares oyentes, y solo una de las alumnas tenía un familiar sordo, en este caso, una hermana.

El contacto con la LSM se dio principalmente al entrar al centro de atención múltiple. Los padres de los alumnos sordos presentan un pobre dominio de la lengua de señas, se comunican con sus hijos con señas caseras, con señas de la LSM y de manera verbal.

\subsection{Desarrollo del taller}

\section{$1^{a}$ etapa: el diagnóstico}

Los alumnos sordos inscritos al taller fueron evaluados con una prueba específica para conocer su nivel competencia del español en su forma escrita. Esta prueba fue diseñada por los estudiantes de la carrera de Letras (coordinadores y ejecutores del taller). A partir de los objetivos señalados en los planes de estudio del sistema de educación básica sobre el nivel de competencia en español escrito, seleccionaron los objetivos conforme a los parámetros que debían tener al encontrarse en este nivel educativo.

La prueba evaluaba la comprensión lectora y la producción escrita, dividida en dos bloques. Un primer bloque correspondía a la identificación de vocabulario para completar las frases, dar sentido a un texto, uso de sinónimos, seguimiento de instrucciones escritas, e identificación de clases de palabras. El segundo consistió en la 
producción de un texto sobre una actividad que les gustara o la narración de lo que habían hecho el día anterior.

De manera general, y desde una perspectiva cualitativa, se pudo observar lo siguiente: a) un déficit en el nivel de vocabulario; b) una inadecuada concordancia entre género y número; c) errores en la flexión verbal; d) errores en la producción de construcciones sintácticas, resultando la mayoría de ellas atípicas; e) no existe una noción de sílaba, produciendo plantillas silábicas anómalas para el caso del español. En cuanto a la producción libre de un texto encontramos una actitud negativa de los jóvenes sordos hacia esta tarea. A la mayoría no les resultó fácil hacer esta actividad, textos escuetos, e incluso varios se rehusaron a hacerla. Los textos que realizaron básicamente fueron de tipo enumerativo, faltaban elementos discursivos para crear un escrito con cohesión.

$2^{a}$ etapa: el diseño y la ejecución

El taller tuvo una duración total de 20 horas con una periodicidad de una vez a la semana. Dada la heterogeneidad en el dominio del español escrito y de la LSM, fue necesario hacer subgrupos. Se agruparon a alumnos sordos con un mayor dominio de LSM con compañeros sordos que no la tuvieran, y de igual manera, se buscó que aquellos alumnos con mayor dominio del español escrito hicieran diadas con aquellos que tuvieran más dificultades.

A partir del marco teórico revisado, se consideró partir de una reflexión sobre la lengua meta (el español). El eje fue el conocimiento y uso de sustantivos, verbos, pronombres y adjetivos. Con este tipo de actividades se buscó la formación de frases nominales y verbales, que permitieran la creación de unidades de mayor alcance. Se hacían juegos de palabras, modelado a través situaciones comunicativas (representaciones). Se propició la reflexión de estas mismas categorías gramaticales en la LSM.

Al inicio de la semana se discutían los contenidos sobre la gramática del español que se abordarían en la sesión del taller. Se determinaba el objetivo de la clase, la secuencia y tipo de actividades, así como la evaluación de la sesión, pero a su vez cada alumno de Letras creaba actividades diferentes para los alumnos sordos que le correspondía monitorear.

Si bien se perseguía el mismo objetivo se hicieron adecuaciones dependiendo de las características de los alumnos. Se realizaba una actividad común de apertura, y otras distintas dependiendo de las necesidades de cada subgrupo, su competencia en lengua de señas o del español, y la reflexión sobre los elementos formales de ambas lenguas. De manera alterna había una persona que podía coordinar el desarrollo del taller en ese día (secuencia de actividades grupales) y cada estudiante oyente coordinaba el trabajo con su propio grupo de alumnos sordos. Al final de la sesión del taller se evaluaba el trabajo de todo el colectivo (sordo y oyente) con el fin de retroalimentar a los miembros del grupo.

\section{$3^{a}$ etapa: evaluación del taller}

En el mes de diciembre de 2014 terminó este taller, y nuestra evaluación de este trabajo la dividimos en dos grandes apartados. Por un lado el reconocimiento del colectivo de alumnos sordos en cuanto a la conformación de un grupo con fuertes lazos de solidaridad, para apoyarse en las tareas y por el interés en que todos aprendieran lo que se exponía en clase. Durante el desarrollo del taller, poco a poco los alumnos sordos que entendían mejor la clase al percatarse que alguno de los compañeros no la entendía se 
ofrecían a explicarla. Esto favoreció no solo a los alumnos sordos sino a los propios compañeros oyentes que aprendían esta forma de explicar la consigna o el contenido que se estaba discutiendo. Así, en un segundo momento, éstos podían explicar de una manera más clara al interior de los subgrupos que coordinaban.

Los logros en cuanto al dominio del español escrito no podemos considerarlos significativos, pues los alumnos sordos continuaron casi con los mismos errores que al inicio del taller. No obstante, tuvieron una mayor conciencia en cuanto a los diferentes patrones silábicos que existen en el español en la formación de palabras. La relación de concordancia de género entre el sustantivo y el artículo. Y, sus producciones libres dejaron pasaron de ser enumerativas a descriptivas.

No obstante, observamos avances significativos en cuanto a la reflexión de la lengua de señas. Los alumnos sordos comenzaron a establecer diferencias entre la estructura gramatical de la LSM y del español, preguntaban sobre la organización sintáctica de la LSM y su traducción al español, así como de manera inversa. Por ejemplo, la función sintáctica de ciertas clases de palabras entre ellas los artículos (que en la LSM no existen); o la morfología verbal que en la legua de señas se realiza en algunos casos con los rasgos no manuales articulados de manera simultánea al producir el verbo. Este análisis les permitio reconocer algunas de las reglas de la lengua escrita.

\section{A manera de conclusión}

Este trabajo constituye un acercamiento hacia la discusión de la literacidad de la comunidad sorda. Por un lado nos permitió reflexionar sobre la necesidad de conjuntar los aportes de las distintas ramas de la lingüística, así como de otras disciplinas de las humanidades y de las neurociencias, con el fin de hacer operativo la propuesta de un modelo bilingüe para la enseñanza del sordo.

Los hallazgos que obtuvimos de este taller apuntan hacia el reconocimiento de la hipótesis léxica; de la importancia de propiciar una reflexión metalingüística en ambas lenguas (LSM y español); y de continuar indagando sobre el papel de la memoria de trabajo en este proceso de enseñanza de la lengua escrita. Asimismo la experiencia vivida nos demuestra que una actitud positiva hacia los usuarios de lenguas y culturas diferentes a las de la mayoría, haría que la escuela fuera un espacio que favoreciera el bilingüismo.

En este taller, los jóvenes sordos y los oyentes conformaron un solo colectivo llamado "Manos a la hoja". Se demostró que el tener una lengua diferente no es un impedimento para comunicarse si estamos abiertos a la diversidad, a aprender uno del otro. Esto nos conduce a su vez a continuar discutiendo la complejidad del desafío de tener dos lenguas en contacto (LSM-español), y dos culturas (oyente y sorda) en un mismo espacio educativo, para implementar una adecuada política lingüística. A reconocer que en el contexto educativo bilingüe enseñar utilizando principalmente una lengua, o ponderar el aprendizaje de aquella que es la de la mayoría, conlleva a una serie de situaciones que requiere de la mirada de la sociolingüística, de la etnografía de la comunicación, y de la pragmalingüística.

Por tanto, habría que destacar que no solo es importante el conocimiento de las reglas de la lengua dominante sino los usos de esta lengua en contextos determinados. Favorecer así el desarrollo de la competencia comunicativa de los alumnos, a partir de identificar 
que hay usuarios de lenguas y culturas diferentes, pero que hoy más que nunca comparten espacios y metas comunes.

\section{Referencias}

Ayora Esteban, M. C. (2010). Diversidad lingüística y cultural en un ámbito educativo de lenguas en contacto. Pragmalingüística, 18, 30-52.

Blanton, R. L., Nunnally, J. C. y Odom, P. B. (1967). Graphemic, phonetic, and associative factors in the verbal behavior of deaf and hearing subjects. Journal of Speech and Hearing Research, 10, 225-231. doi:10.1044/jshr.1002.225

Charlier, B. L. y Leybaert, J. (2000). The rhyming skills of deaf children educated with phonetically augmented speech reading. Quarterly Journal of Experimental Psychology, 53, 349-375. doi:10.1080/713755898

Coronado Suzán, G. (1997). Espacios para el billingüismo. Entre la imposición estatal y la apropiación comunal. En B. Garza Cuarón (Coord.), Políticas lingüísticas en México (pp. 135-150) México: UNAM.

Ferreiro, E. (1997). El bilingüismo: una visión positiva. En B. Garza Cuarón (Coord.), Políticas lingüísticas en México (pp. 293-302). México: UNAM.

Hamel, R. E. (2003). El papel de la lengua materna en la enseñanza: particularidades en la educación bilingüe. En I. Jung y L. E. López (Eds.), Abriendo la escuela: lingüística aplicada a la enseñanza de lenguas (pp. 248-260). Madrid: Morata.

Hamel, R. E. (2008). Bilingual education for indigenous communities in Mexico. En J. Cummins y N. Hornberger (Eds.), Encyclopedia of language and education (pp. 311-322). Nueva York: Springer.

Hanson, V. (1989). Phonology and reading: evidence from profoundly deaf readers. En D. Shankweiler e I. Y. Liberman (Eds.), Phonology and reading disability, solving the reading. puzzle (pp. 69-89). Ann Arbor, MI: The University of Michigan Press.

Howard, D. y Patterson, K. E. (1992). The pyramids and palm trees test. Londres: Thames Valley Test Company.

INEGI. (2013). Las personas con discapacidad en México. Una visión al 2010. México: Instituto Nacional de Estadística, Geografía e Informática.

Kavanagh, J. I. (Ed.). (1968). Communicating by language: the reading process. Bethesda, MD: Department of Health, Education and Welfare.

Kavanagh, J. I. y Mattingly, G. (1972). Language by ear and by eye: the relationships between speech and reading. Boston, MA: MIT Press.

Lissi, M. R., Svartholm, K. y González, M. (2012). El enfoque bilingüe en la educación de los sordos: sus implicancias para la enseñanza y aprendizaje de la lengua escrita. Estudios Pedagógicos, 38(2), 299 -320. doi:10.4067/s07 18-07052012000200019

Locke, J. L. (1978). Phonemic effects in the silent reading. Cognition, 6, 175-187. doi:10.1016/0010-0277(78)90012-4

Mayer C. y Akamatsu, C. T. (2003). "Bilingualism and Literacy”. En M. Marschark y P. E. Spencer (Eds.), Oxford handbook of Deaf studies, languages and education (pp.136-147). Nueva York: Oxford University Press. 
Miller, P. (2006). What the processing of real words and pseudo- homophones tell about thedevelopment of orthographic knowledge in prelingually deafened individuals. Journal of Deaf Studies and Deaf Education, 11, 21-38.

Miller, P. (2010). Phonological, ortographic, and syntactic awareness and theri relation to reading comprehension in prelingually deaf indivuduals: what can we learn from skilled readers? Journal of Development and Physical Disabilities, 22, 549-580. doi:10.1007/s10882010-9195-Z

Miller, P. (2013). Similarities and differences in the processing of written test by skilled and less skilled readers with prelingual deafnes. The Journal of Special Education, 46, 233-244. doi: $10.1177 / 0022466910386790$

Milner, B. (1971). Interhemispheric differences in the localization of psychological processes in man. British Medical Bulletin, 27, 272-277.

Monreal, T. S. y Hernández, S. R. (2005). Reading levels of Spanish deaf students. American Annals of the Deaf, 150(4), 379-387. doi:10.1353/aad.2005.0043

Muñoz-Cruz, H. (2010). Reflexividad sociolingüística de hablantes de lenguas indígenas: concepciones y cambios. México: Universidad Autónoma Metropolitana

OCDE. (2013) Education at glance. Indicators. París: OECD.

Osterrieth, P. A. (1944). Le test de copie d'une figure complexe. Archives de Psychologie, 30, 206356.

Paradis, J., Genesee, F. y Crago, M. B. (2011). Dual language development E゚ disorders. A handbook on bilingualism E' second language learning. Londres: Paul Brookes.

Peña-Casanova, J. (1991). Programa integrado de exploración neuropsicologica. Test Barcelona. Barcelona: Masson.

Pietrosemoli, L. G. (2007). La lectura y la escritura en el sordo: lo qué habría que replantear. Bogotá: Universidad de Los Andes

Rey, A. (1941). L'examen psychologique dans les cas d'encephalopathy traumatique. Archives de Psycholigie, 28, 286-340.

Schirmer, B. (2000). Language and literacy development in children who are deaf. Boston, MA: Allyn \& Bacon.

Schirmer, B. y Williams, C. (2003). Approaches to teaching reading. En M. Marschark y P. E. Spencer (Eds.), Oxford handbook of deaf studies, languages and education (pp. 253-287). Nueva York: Oxford University Press.

Terborg R. y García Landa, L. (2006). Los retos de la planificación del lenguaje en el siglo XXI. México: UNAM.

\section{Breve CV de los autores}

\section{Miroslava Cruz-Aldrete}

Licenciada en Educación Especial en Audición y Lenguaje por la Escuela Normal de Especialización (1993), tiene estudios de Neuropsicología Infantil por la Benemérita Universidad Autónoma de Puebla y la Facultad de Estudios Superiores Zaragoza, UNAM. Doctora en Lingüística por El Colegio de México (2008) con la tesis Gramática 
de la Lengua de Señas Mexicana Es miembro del Sistema Nacional de Investigadores (SNI Nivel I), y del Sistema Estatal de Investigadores (SEI). Colabora en varios proyectos nacionales e internacionales sobre educación y atención a la comunidad sorda señante. Email: miroslava.cruza@uaem.edu.mx

\section{Miguel Ángel Villa Rodríguez}

Licenciado en psicología, UNAM, maestría en neuropsicología, Universidad Autónoma de Barcelona y doctor en psicología de la Salud, Universidad de Guadalajara. Profesor titular "C" definitivo adscrito a la División de Estudios de posgrado e investigación de la FES Zaragoza, UNAM. Líneas de investigación: Neuropsicología del envejecimiento normal y patológico. Organización cerebral de las lenguas de señas. Email: mavilla@unam.mx 